band ligation \pm non-selective ß-blockers. Carvedilol is a vasodilating non-selective B-blocker with alpha-1 receptor and calcium channel antagonism. It has a greater portal hypotensive effect than propranolol and has been shown to be effective in the prevention of a first variceal bleed. Our aim was to compare oral carvedilol with band ligation in the prevention of rebleeding following a first variceal bleed. Methods Patients who were stable 5 days after presentation with a first variceal haemorrhage and had not been taking (or had contraindications to) ß-blockers, were randomised to oral carvedilol (6.25 mg daily then $12.5 \mathrm{mg}$ daily after one week if tolerated) or a band ligation programme. Patients were followed up at clinic after one week, monthly, then 3-monthly. The primary end-point was variceal rebleeding, on intention-to-treat analysis.

Results 63 patients were randomised, 32 to carvedilol and 31 to banding. Fifty-six (89\%) patients had alcohol related liver disease. There was no difference in baseline mean age (51 yrs \pm 10.9 and 50 yrs \pm 13.0 ) or median Childs Pugh score (9, IOR 6-11 and 9, IOR 8-11) for patients randomised to carvedilol or banding respectively. Mean follow-up was 29 months. Compliance was $72 \%$ and $90 \%$ for carvedilol and banding respectively $(p=0.14)$ and there was no difference in the number of serious adverse events between the two groups. Variceal rebleeding occurred during follow-up in 12 (37.5\%) and $9(29.0 \%)$ patients in the carvedilol and banding groups respectively $(p=0.72$ ), with mortality $25.0 \%$ and $51.6 \%$ respectively $(p=0.058)$. The differences in outcome the between groups were similar using per protocol analysis. This interim analysis indicates that to show a significant difference in rebleeding, 482 patients would be required in each group.

Conclusion Carvedilol is not clearly superior to band ligation in the prevention of variceal rebleeding. However there appears to be a survival benefit for patients taking this drug compared with those undergoing banding, which requires further exploration.

Disclosure of Interest None Declared

\section{OC-004 IN VITRO STEROID SENSITIVITY ACCURATELY PREDICTS 6 MONTH MORTALITY IN PATIENTS WITH SEVERE ALCOHOLIC HEPATITIS}

doi:10.1136/gutjnl-2013-304907.004

\begin{abstract}
1,2, A Dhanda, ${ }^{3} \mathrm{~A}$ Di Mambro, ${ }^{2} \mathrm{~A}$ McCune, ${ }^{2} \mathrm{~F}$ Gordon, ${ }^{2} \mathrm{~J}$ Portal, ${ }^{2} \mathrm{P}$ Collins. ${ }^{1}$ School of Clinical Sciences, University of Bristol; ' 2 Department of Liver Medicine, University Hospitals Bristol NHS Foundation Trust, Bristol; ${ }^{3}$ Department of Gastroenterology, Weston General Hospital, Weston-super-mare, UK
\end{abstract}

Introduction Severe alcoholic hepatitis (SAH) has a high mortality especially in those who fail to respond to steroid treatment. Early identification of steroid resistant patients may allow rapid implementation of other therapies, which may improve patient outcome. An early change in bilirubin has prognostic value but requires 7 days of steroid treatment first. Our group have previously reported that a simple $48 \mathrm{~h}$ in vitro method for assessing steroid sensitivity (dexamethasone inhibition of lymphocyte proliferation; DILPA) correlates with 6 month mortality in patients with SAH[1]. We aimed to determine the accuracy of the DILPA in predicting outcome and to compare it to existing models of prognosis in SAH.

Methods Peripheral blood was drawn from consecutive patients with a clinical diagnosis of SAH (Maddrey discriminant function $[\mathrm{MDF}]>32$ ). All subjects gave informed consent and ethical approval was obtained from the regional ethics committee. The primary outcome measure was 6 month mortality. Leukocytes, isolated by density gradient centrifugation, were cultured for $48 \mathrm{~h}$ with the mitogen PHA in the presence or absence of dexamethasone. Tritiated thymidine was added for the final $6 \mathrm{~h}$ of culture before proliferation was measured on a beta counter. Maximum suppression of lymphocyte proliferation by dexamethasone was calculated (Imax).
An Imax of $<60 \%$ indicates in vitro steroid resistance as previously determined. The accuracy of the DILPA and existing scoring systems (MDF, MELD, Glasgow and Lille scores) was calculated by area under the receiver operating characteristic (AUROC). Logistic regression of baseline and day 7 parameters was performed to determine independent predictors of outcome.

Results 43 patients were recruited (21\% female, median age 45 ). Patients who survived 6 months had a significantly higher Imax than those who didn't (79.2\% v 41.7\%; $p=0.0003)$. An Imax of $>$ $60 \%$ had a $88 \%$ sensitivity and $78 \%$ specificity in predicting 6 month survival and an AUROC of 0.83. MDF, MELD, Glasgow and Lille scores all had lower AUROC values for predicting 6 months survival than $\operatorname{Imax}(0.36,0.4,0.49$ and 0.61 respectively). A stepwise logistic regression demonstrated that only Imax, Day 0 albumin and change in bilirubin at Day 7 of treatment were independent predictors of 6 month survival.

Conclusion The DILPA provides a simple, fast and accurate in vitro method of predicting clinical outcome in patients with SAH. This could be applied in clinical practise to identify patients who will not respond to steroid therapy within 2 days of starting treatment.

Disclosure of Interest None Declared

\section{REFERENCE}

1. Di Mambro et al. Hepatology 2011;53:1316-22.

\section{OC-005 THE EARLY EXPANSION OF INTRAHEPATIC NK CELLS IS ASSOCIATED WITH CLEARANCE OF HCV AFTER IFN-ALPHA -BASED TREATMENT}

doi:10.1136/gutjnl-2013-304907.005

1.,T Pembroke, ' $A$ Gallimore, ${ }^{1} A$ Godkin. ${ }^{1}$ School of Medicine, Cardiff University, Cardiff, UK

Introduction Chronic hepatitis $\mathrm{C}$ virus (HCV) infection is a major cause of liver failure, cirrhosis and hepatocellular carcinoma. Current treatments are evolving but still largely rely on IFN $\alpha$ in combination with ribavirin $+/$ - protease inhibitors. IFNa can activate Natural killer (NK) cells: large granular lymphocytes that can target virally-infected cells. NK cells may play a role in the immune response to chronic HCV. We examined whether the kinetics of NK cell expansion and activation affected long-term treatment success. Methods Repeated matched blood and intrahepatic (IH) NK cells were obtained from 9 patients before treatment and on days 1, 3, 7, 14, 28 and 86 of treatment. IH samples were obtained by fine needle aspirations. NK cell phenotype (CD16, NKp30, NKp46 \& NKG2D) and functional markers (CD107a, IFNg \& granzyme B) were assessed by flow cytometry. Rate of viral clearance ( $k$ ) was calculated from serial serum viral loads during treatment.

Results Multiple IH samples were obtained from 9 subjects. 5 of the 9 patients achieved viral clearance i.e. SVR; 4 failed treatment. The proportion of IH lymphocytes that were NK cells increased from mean $10 \%(+/-0.97 \%$ SEM) at baseline to $16.1 \%(+/-2.43 \%$ SEM) after 24 hours $(p=0.021)$ and $17.9 \%(+/-4.75 \%$ SEM $)$ after 72 hours $(p=0.023)$. The proportions returned to baseline levels from day 7 onwards. The proportion of IH NK cells was greater in patients who achieved SVR (18.6\%) than those who failed treatment $(11.9 \%)$. The rate of viral clearance correlates with IH NK cells at day 1 ( $p=0.036)$. IH NK cells at 24 hours demonstrated an increase in activation i.e. increase CD107a externalisation, decreased granzyme $\mathrm{B}$ and $\mathrm{CD} 16$ expression.

Conclusion IH NK cells are cytotoxically active during the early phase of treatment when reduction in viral load is most pronounced. The rate of expansion of these activated NK cells in the first 24 hours indicates treatment success or failure. These findings suggest that strategies to improve early NK cell migration to the liver during treatment may lead to better treatment outcomes.

Disclosure of Interest None Declared 